\title{
Vertical distribution of chromium in the atmospheres of HgMn stars ${ }^{\star}$
}

\author{
I. Savanov ${ }^{1,2}$ and S. Hubrig ${ }^{3}$ \\ 1 Astrophysical Institute Potsdam (AIP), An der Sternwarte 16, 14482 Potsdam, Germany \\ 2 Crimean Astrophysical Observatory, Nauchny, Crimea, Ukraine and Isaac Newton Institute of Chile, Crimean Branch \\ 3 European Southern Observatory, Casilla 19001, Santiago 19, Chile \\ e-mail: shubrig@eso.org
}

Received 18 June 2003 / Accepted 5 August 2003

\begin{abstract}
We use multiplet $30 \mathrm{Cr}$ II lines in the wings of $\mathrm{H}_{\beta}$ to test the hypothesis of an anomalous concentration of $\mathrm{Cr}$ in the upper layers of the atmospheres of a sample of $10 \mathrm{HgMn}$ stars. These lines are at different distances from the $\mathrm{H}_{\beta}$ line center and are therefore formed at different depths in the stellar atmosphere. Except for HD 49606, all HgMn stars show an increase in $\mathrm{Cr}$ abundance with height in the stellar atmosphere. A similar vertical distribution of $\mathrm{Cr}$, but less pronounced, has been previously found in Am stars. In contrast, no variation of $\mathrm{Cr}$ abundance with the depth has been found for the normal late B-type star HD 196426 and the weak magnetic late B-type star HD 168733. It is possible that in HgMn stars the vertical stratification parameter, $a$, depends on $T_{\text {eff }}$, with the strongest vertical gradient being found in the hotter stars. No correlation was found between $a$ and the average stellar abundance $\log \varepsilon(\mathrm{Cr} / \mathrm{H})$.
\end{abstract}

Key words. stars: abundances - stars: atmospheres - stars: chemically peculiar

\section{Introduction}

The HgMn stars belong to spectral types between A0 and B7 and show marked peculiarities in atmospheric abundances. The most distinctive features of their atmospheres are extreme overabundances of $\mathrm{Hg}$ (sometimes exceeding $5 \mathrm{dex}$ ) and/or Mn (up to 3 dex). The HgMn stars are slow rotators (Abt et al. 1972) and appear not to have strong large-scale organized magnetic fields such as those of other chemically peculiar (CP) stars of similar temperature, the $\mathrm{Si}$ and He-weak stars. This does not definitely rule out the presence of more complex fields of kiloGauss order, as suggested by Hubrig and collaborators (Hubrig \& Castelli 2001 and references therein).

To explain the abundance anomalies in the outer layers of $\mathrm{HgMn}$ stars, radiatively driven selective diffusion in relatively quiescent atmospheres is most often invoked. The $\mathrm{HgMn}$ stars are too hot to have a superficial hydrogen convection zone, and, due to gravitational settling of He they are expected not to have $\mathrm{He}^{+}$convective zones. Therefore, diffusion occurs in the absence of mixing due to convection and of stronger meridional circulations that will occur in faster rotators.

In contrast to the $\mathrm{CP}$ stars with large-scale organized magnetic fields, the $\mathrm{HgMn}$ stars generally do not exhibit periodic

Send offprint requests to: I. Savanov, e-mail: isavanov@aip.de

* Based on observations obtained at the European Southern Observatory, La Silla and Paranal, Chile (ESO programmes Nos. 62.L-0348, 65.I-0644, 67.D-0579). variations in photometry and spectral line intensities. The interpretation of variability of magnetic $\mathrm{CP}$ stars is that the surface properties (e.g. abundance distribution of certain elements, distribution of the magnetic field) are non-uniform and nonsymmetric with respect to their rotation axis. On the basis of indirect arguments, Hubrig \& Mathys (1995) had suggested that at least two chemical elements ( $\mathrm{Hg}$ and $\mathrm{Mn})$ might be inhomogeneously distributed over the surface of $\mathrm{HgMn}$ stars. Very recently, for the moderately rotating $\operatorname{HgMn}$ star $\alpha$ And periodic line profile changes in the $\mathrm{Hg}$ II $\lambda 3984$ have been reported by Wahlgren et al. (2001) and Adelman et al. (2002), who could show that the reason for the variability is the non-symmetrical distribution of $\mathrm{Hg}$ over the surface of $\alpha$ And.

Aside from surface inhomogeneities in the atmospheres of CP stars, an observational evidence is accumulating in the last years that a number of observed spectroscopic features cannot be reproduced with the standard model atmosphere codes in which vertical abundance variations are neglected and where a homogeneous photospheric abundance is assumed (e.g. Ryabchikova et al. 2002). Most observational studies of the abundance stratification have been devoted to magnetic CP stars and rapidly oscillating Ap stars (e.g. Ryabchikova et al. 2003), for which the impact of the abundance stratification could be significant and for which it has to be taken into account in modelling of pulsational radial velocities, magnetic fields and for abundance determination. 
A direct method of determining the vertical stratification is by a comparative analysis of spectral lines which are formed at different depths. Abundances from lines of the same ions formed on either side of the Balmer jump, in the UV and visual spectral regions (e.g., Alecian 1982; Lanz et al. 1993), or from the $\mathrm{Cr}$ II lines of mult. 30 in the wings of $\mathrm{H}_{\beta}$ (Savanov et al. 2001a), have been determined in a small number of CP stars. The method of abundance analysis using eight $\mathrm{Cr}$ II lines of the same multiplet in the wings of the $\mathrm{H}_{\beta}$ line was introduced by Khokhlova \& Topil'skaya (1992). This presents an excellent opportunity to investigate the vertical stratification in normal and chemically peculiar stars of spectral types from $\mathrm{B}$ to $\mathrm{F}$. The $\mathrm{Cr}$ II lines have well-determined relative oscillator strengths and cover a fairly wide wavelength range in the wings of the $\mathrm{H}_{\beta}$ line, being situated at distances $\Delta \lambda$ of $1.1 \AA$ to $49.0 \AA$ from the line center. If stratification of $\mathrm{Cr}$ is indeed present, and if this element is overabundant in a thin surface layer, then the dependence of the spectral line intensities on $\Delta \lambda$ will be markedly different from that in a homogeneous atmosphere. A review of the methods of studying vertical stratification in the stellar atmospheres is given in Savanov \& Kochukhov (1998).

Usually, the abundance anomalies are ascribed to hydrodynamical processes in the outer stellar layers - radiatively-driven diffusion, aided or mitigated by magnetic fields, weak (possibly anisotropic) stellar winds, turbulence, and rotational mixing. However, it is difficult to determine the mechanisms responsible for abundance anomalies in the absence of accurate observational information about elemental abundances. The vertical stratification of $\mathrm{Cr}$ has not yet been studied for a representative number of HgMn stars. Savanov et al. (2001a) studied Cr II lines of mult. 30 in the wings of the $\mathrm{H}_{\beta}$ line in a sample of magnetic CP stars, Am stars and one HgMn star (46 Dra). We present in this paper our results of the analysis of abundance stratification in ten slowly rotating $\mathrm{HgMn}$ stars. One weak magnetic late B-type star, HD 168733, and one normal late B-type star, HD 196426, have been observed as comparison stars.

\section{Observations and data reduction}

Spectra of seven HgMn stars in our sample, HD 33904, HD 49606, HD 71066, HD 78316, HD 110073, HD 124740 and HD 165493, were observed on March 14, 1999 at ESO with the New Technology Telescope (NTT) and the ESO Multi Mode Instrument (EMMI). The red arm of EMMI was used in the cross-dispersed echelle mode. The main dispersing element was the EMMI grating \#14, a 31.6 lines/mm echelle grating mounted in the R4 configuration. As cross-disperser, the EMMI grism \#5 was employed. The detector was CCD \#36, a Tektronix with $2048 \times 2048$ pixels of $24 \times 24 \mu \mathrm{m}^{2}$. Setting the entrance slit of the spectrograph to a width of 0 '. 8 , a resolving power $\lambda / \Delta \lambda \approx 7 \times 10^{4}$ was achieved over the whole wavelength range (3970-6620 $)$.

The data reduction was performed using the ESO image processing package MIDAS. Echelle orders were automatically detected by a Hough transform, and a two-dimensional polynomial fit was computed to define their location on the CCD. For both the scientific and the flat field frames, the background scattered light was modelled by fitting its level in the interorders by
Table 1. Model atmosphere parameters of the studied stars.

\begin{tabular}{|c|c|c|c|c|}
\hline Star & $T_{\text {eff }}$ & $\log g$ & $\begin{array}{l}V \sin i \\
\mathrm{~km} \mathrm{~s}^{-1}\end{array}$ & Reference \\
\hline HD $1909^{a}$ & 12400 & 4.00 & 13.0 & Adelman et al. (1996) \\
\hline HD 33904 & 12500 & 3.62 & 15.0 & Adelman et al. (1996) \\
\hline HD 49606 & 14375 & 3.90 & 15.0 & Adelman et al. (1996) \\
\hline HD 71066 & 12100 & 3.95 & 2.0 & Hubrig et al. (1999) \\
\hline HD $78316^{b}$ & 13250 & 3.75 & 6.0 & Adelman \& Pintado (2000) \\
\hline HD 110073 & 12900 & 3.75 & 1.8 & Woolf \& Lambert (1999) \\
\hline HD $124740^{c}$ & 10350 & 4.00 & 2.0 & Dolk et al. (2003) \\
\hline HD 165493 & 13890 & 3.90 & 2.8 & Hubrig et al. (1999) \\
\hline HD 168733 & 13500 & 3.30 & 10.0 & Lanz et al. (1993) \\
\hline HD 175640 & 12000 & 3.95 & 2.5 & Hubrig et al. (1999) \\
\hline HD 178065 & 12200 & 3.54 & 1.5 & Hubrig et al. (1999) \\
\hline HD 196426 & 12815 & 3.89 & 3.0 & Hubrig et al. (1999) \\
\hline
\end{tabular}

$a$ For HD 1909B: $T_{\text {eff }}=9000 \mathrm{~K}, \log g=4.0, V \sin i=12 \mathrm{~km} \mathrm{~s}^{-1}$ (Wahlgren et al. 2002)

$b$ For HD 78316B: $T_{\text {eff }}=8000 \mathrm{~K}, \log g=4.0, V \sin i=40 \mathrm{~km} \mathrm{~s}^{-1}$ (Ryabchikova et al. 1998)

$c$ For HD 124740B: $T_{\text {eff }}=8000 \mathrm{~K}, \log g=4.0, V \sin i=5 \mathrm{~km} \mathrm{~s}^{-1}$ (Dolk et al. 2003)

a 2-dimensional cubic spline. The result was subtracted from the corresponding exposure, prior to the division of the scientific frame by the flat field. The echelle orders were then extracted from the resulting frames.

Spectra of the three HgMn stars HD 1909, HD 175640 and HD 178065, have been recorded on June 13, 2001 at ESO with the VLT UV-Visual Echelle Spectrograph UVES at UT2. We used the UVES Dichroic standard settings covering the spectral range from $3030 \AA$ to $10000 \AA$. The slit width was set to 0.3 , corresponding to a resolving power of $\lambda / \Delta \lambda \approx 1.1 \times 10^{5}$. One weak magnetic late B-type star, HD 168733, and one normal late B-type star, HD 196426, have been observed with the UVES standard setting RED 580 covering the spectral range from $4800 \AA$ to $6800 \AA$ and the entrance slit of the spectrograph set to 0.3 on May 28, 2000. The spectra have been reduced by the UVES pipeline Data Reduction Software (version 1.4.0), which is an evolved version of the ECHELLE context of MIDAS. The signal-to-noise ratios of the resulting NTT and UVES spectra are very high, ranging from 200 to 500 per pixel in the one-dimensional spectrum.

\section{Spectrum synthesis calculations}

Model atmosphere parameters for the studied HgMn stars have already been determined in the past by us and other authors. In Table 1, for each star, Cols. 2-4 list effective temperature, surface gravity and the $v \sin i$ values. The last column gives references to the papers in which the model atmosphere parameters have been determined. Three stars in our sample, HD 1909, HD 78316 and HD 124740, are known to be spectroscopic binaries. The adopted parameters of the companions are presented in the footnotes of Table 1. Model atmospheres were taken from the Kurucz model grid apo0k2odfnew (Kurucz 2000). 
Table 2. The $\mathrm{Cr}$ abundances derived from the individual $\mathrm{Cr}$ II lines of mult. 30 .

\begin{tabular}{|c|c|c|c|c|c|c|c|c|c|c|c|c|c|c|}
\hline $\begin{array}{l}\lambda \\
\AA\end{array}$ & $\begin{array}{c}\Delta \lambda \\
\AA\end{array}$ & $\begin{array}{r}\log g f \\
(V A L D)\end{array}$ & $\begin{array}{l}\text { HD } \\
1909\end{array}$ & $\begin{array}{c}\text { HD } \\
33904\end{array}$ & $\begin{array}{c}\mathrm{HD} \\
49606\end{array}$ & $\begin{array}{c}\mathrm{HD} \\
71066\end{array}$ & $\begin{array}{c}\mathrm{HD} \\
78316\end{array}$ & $\begin{array}{c}\text { HD } \\
110073\end{array}$ & $\begin{array}{c}\text { HD } \\
124740\end{array}$ & $\begin{array}{c}\mathrm{HD} \\
165493\end{array}$ & $\begin{array}{c}\mathrm{HD} \\
168733\end{array}$ & $\begin{array}{c}\text { HD } \\
175640\end{array}$ & $\begin{array}{c}\text { HD } \\
178065\end{array}$ & $\begin{array}{c}\text { HD } \\
196426\end{array}$ \\
\hline 12 & 48 & -1.96 & -6.40 & -6.00 & -6.20 & -6.30 & -6.30 & -5.70 & -5 . & -6.90 & -5.60 & -5.60 & -6.05 & -6.65 \\
\hline 4.1 & .15 & -0.97 & -6.40 & -5.85 & -6.15 & -6.10 & -6.30 & -5.60 & -5 & -6.90 & -5.70 & -5.55 & -5.95 & -6.70 \\
\hline 6.23 & 25.09 & -1.96 & -6.20 & -5.90 & -6.20 & -6.20 & -6.20 & -5.65 & -5.80 & -6.60 & -5.50 & -5.55 & -5.95 & -6.70 \\
\hline 4848.24 & 13.08 & -1.15 & -6.40 & -5.80 & -6.25 & -6.20 & -6.10 & -5.65 & -5.10 & -6.60 & -5.60 & -5.35 & -5.85 & -6.80 \\
\hline 4856.19 & 5.13 & -2.14 & -6.00 & -5.80 & & -5.80 & -6.00 & -5.40 & -5.50 & & -5.50 & -5.30 & -5.80 & -6.60 \\
\hline 4864.33 & 2.98 & -1.36 & -6.00 & -5.70 & & -5.75 & -6.00 & -5.40 & -5.10 & -6.30 & -5.70 & -5.00 & -5.70 & -6.60 \\
\hline 4876.40 & 15.08 & -1.46 & -6.30 & -5.80 & & -6.20 & -5.90 & -5.60 & -5.70 & -6.30 & -5.65 & -5.40 & -5.95 & -6.75 \\
\hline 4884.61 & 23.29 & -2.10 & -6.10 & -5.90 & -6.25 & -6.30 & -6.00 & -5.70 & -5.80 & -6.90 & -5.55 & -5.60 & -6.00 & -6.60 \\
\hline
\end{tabular}

The spectra were analysed using the spectral synthesis code STARSP (Tsymbal 1996). The details concerning the calculation of synthetical spectra, broadening due to the instrumental profile and stellar rotation, and fitting to the observations can be found in the paper of Savanov et al. (2001a). The Vienna Atomic Line Database VALD (Piskunov et al. 1995) was used as a primary source of the oscillator strengths and other spectral line parameters. The synthetic spectra of three $\mathrm{HgMn}$ stars which are known to be spectroscopic binaries, HD 1909, HD 78316 and HD 124740, were calculated and combined using the BINARY routine in STARSP, or with an IDL routine based on general relations for composite spectra in binary stars which can be found in Savanov et al. (2001a).

Adelman (1994) showed that most HgMn stars have little or no turbulence. Therefore, in all calculations we assumed zero microturbulent velocity. The $v \sin i$ values have been taken either from the referred papers or they were estimated independently from the comparison of the observed and computed spectra, after having degraded the computed spectra for instrumental broadening. An abundance analysis of $\mathrm{Cr}$ II was made by matching the observed line profiles and computed synthetic spectra. As all eight $\mathrm{Cr}$ II lines of the mult. 30 lie in the wings of the hydrogen $\mathrm{H}_{\beta}$ line, particular attention has been paid to drawing the continuum. The adopted continuum completely relied on the synthetic $\mathrm{H}_{\beta}$ profiles, so that the continuum level for the observed spectra was fixed by the computed synthetic spectra in which the wings of the $\mathrm{H}_{\beta}$ line profile were regarded as local continuum. To check the correctness of our results, the synthetic spectra have also been calculated with two other different codes, SYNT (Piskunov 1992) and SPECTRUM (Gray http://www1.appstate.edu/). The results of the $\mathrm{Cr}$ II abundances are in good agreement between all three codes. The difference in synthetic hydrogen line profiles calculated by these codes becomes noticeable only in the central part $\left( \pm 2 \AA\right.$ from the center of $\left.\mathrm{H}_{\beta}\right)$. However, this part of the line profile is not used in our abundance analysis.

In the previous analyses of $\beta \mathrm{CrB}$ by Savanov $\&$ Kochukhov (1998) and of 17 Com by Savanov et al. (2001a) the authors studied the dependence of the accuracy of the $\mathrm{Cr}$ abundance determination on the changes in the stellar parameters and metallicity by running multiple syntheses. They could show that the small changes in local continuum due to errors in atmospheric parameters or incorporating of additional broadening of the $\mathrm{H}_{\beta}$ profile do not produce any significant effect on the determinations of parameters characterizing the $\mathrm{Cr}$ vertical stratification. The atomic data for the synthesis of $\mathrm{Cr}$ II lines of the 30th multiplet used in these previous studies were taken from the VALD database. The theoretical $g f$-values given by Kurucz \& Bell (1995) supported the relative scale of oscillator strengths. In addition, the calculations for the standard stars with no stratification of $\mathrm{Cr}$ in their atmospheres have been regarded as a check for reliability of the procedure of the analysis and of the $g f$-values.

To estimate the uncertainties in the resulting values of $\log \varepsilon(\mathrm{Cr} / \mathrm{H})$ and the accuracy of the fitting of the observed spectrum by the synthetic spectrum, a grid of synthetical spectra has been calculated for each star assuming a change of $\pm 0.30 \mathrm{dex}$ in the $\mathrm{Cr}$ abundance. The values of $\log \varepsilon(\mathrm{Cr} / \mathrm{H})$ for each $\mathrm{Cr}$ II line have been obtained by quadratic interpolation in the grid. As a result, the uncertainty in the fitting procedure introduces an error of about $0.05 \mathrm{dex}$ in $\log \varepsilon(\mathrm{Cr} / \mathrm{H})$ for the $\mathrm{HgMn}$ stars with the highest $\mathrm{Cr}$ abundance. The error is by a factor 1.5-2.0 larger for the hot $\mathrm{HgMn}$ stars where the $\mathrm{Cr}$ II lines are intrinsically weak or $\mathrm{Cr}$ is underabundant. The results for the $\mathrm{Cr}$ abundances of the studied stars derived from different $\mathrm{Cr}$ II lines of mult. 30 are presented in Table 2. For the two hottest $\mathrm{HgMn}$ stars, HD 49606 and HD 165493, a few of the Cr II lines which are the closest to the center of the $\mathrm{H}_{\beta}$ line are very weak and could not be used for the abundance analysis.

\section{Results and discussion}

Figure 1 shows plots of the $\mathrm{Cr}$ abundances as a function of distance from $\mathrm{H}_{\beta}$ line center for all 12 stars of our sample including the components of the $\mathrm{HgMn}$ spectroscopic binary 46 Dra from Savanov et al. (2001a). Abundances are shown on a scale where $\log \varepsilon(\mathrm{H})=0.0$. The results of our analysis are summarized in Table 3. In the second column we give the linear regression coefficient $a$ (the average $\mathrm{Cr}$ abundance gradient) and its error $\sigma_{a}$ found in an approximation of the $\mathrm{Cr}$ abundance as a function of $\Delta \lambda$ by the formula $\log \varepsilon(\mathrm{Cr} / \mathrm{H})=a \times \Delta \lambda+b$. The coefficient $a$ is equal to the tangent of the angle of inclination of the dependence of $\log \varepsilon(\mathrm{Cr} / \mathrm{H})$ on $\Delta \lambda$ and is a quantitative characteristic of the vertical $\mathrm{Cr}$ abundance gradient. Negative values of $a$ correspond to an increase of chromium abundance in upper layers of the stellar atmosphere. The linear regression coefficient, $a$, and its error, $\sigma_{a}$, have been determined with an IDL routine which uses the subroutine SVDFIT. This parameter can 

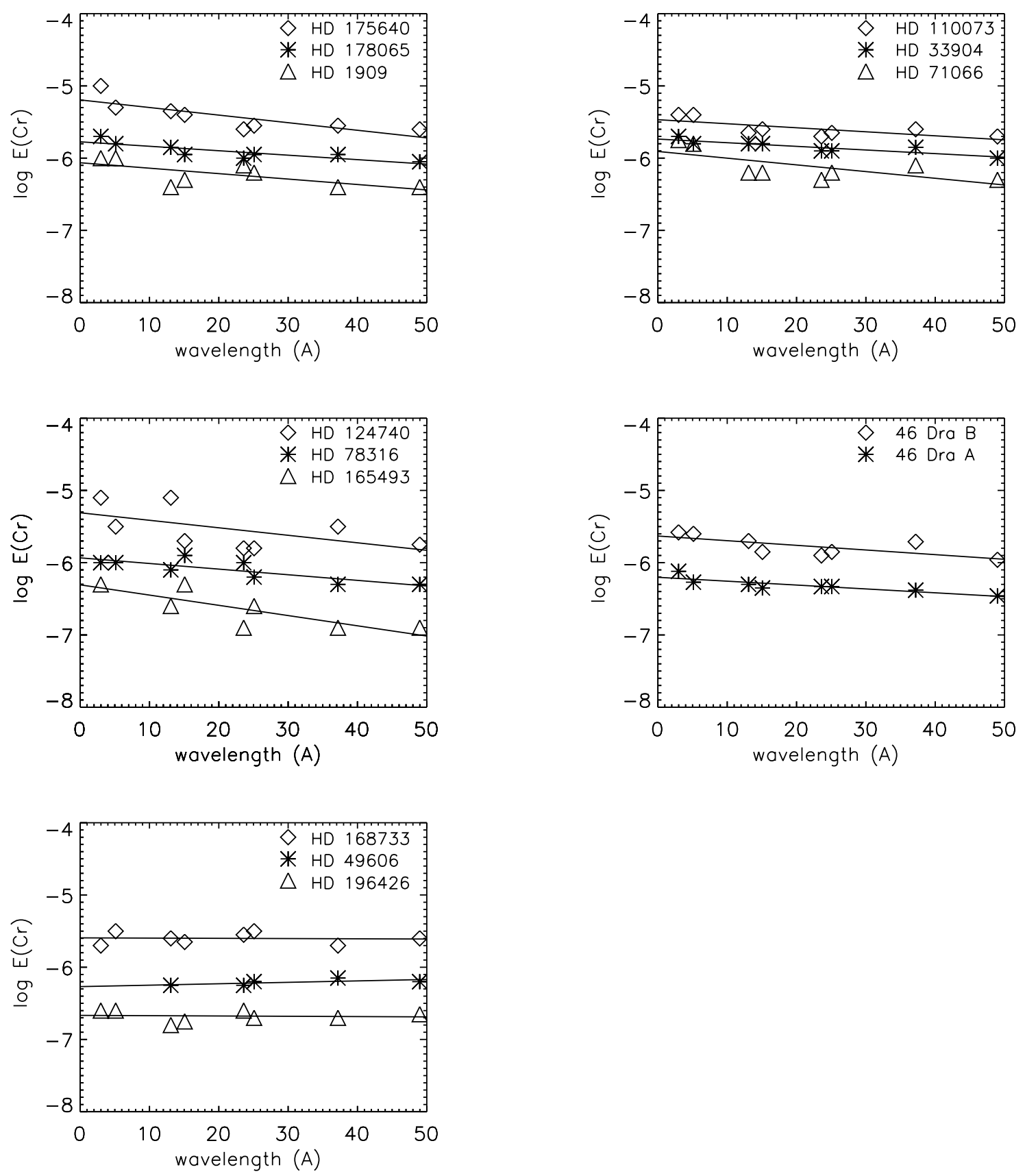

Fig. 1. Linear approximation of the dependence of the $\mathrm{Cr}$ abundance on the distance from the center of the $\mathrm{H}_{\beta}$ line.

be regarded as a first approximation to the vertical distribution of the element in the stellar atmosphere until modeling of concentration of atoms with optical or geometrical depth can be performed (e.g. Savanov et al. 2001b; Ryabchikova et al. 2002). The last column of Table 3 shows the "mean" values of the $\mathrm{Cr}$ abundances which were determined from the $\log \varepsilon(\mathrm{Cr} / \mathrm{H})$ of the four $\mathrm{Cr}$ II lines at distances larger than $20 \AA$ from the center of the $\mathrm{H}_{\beta}$ line and should be regarded as upper limits for the $\mathrm{Cr}$ abundances.

In Table 4 we compare the "mean" values of the $\mathrm{Cr}$ abundance with the results obtained in previous studies. The agreement between our abundances and the abundances given in the literature is rather good, although some discrepancies exist for the stars HD 1909, HD 49606 and HD 78316. The binary nature of HD 1909 has been recognized by Wahlgren et al. (2002). However, the star has been considered as a single star in the studies of Guthrie (1984) and Adelman et al. (1996) who give lower values of $\mathrm{Cr}$ abundance compared to our determination. For HD 49606, Adelman et al. (1996) determined a higher $\mathrm{Cr}$ abundance $(-5.89)$. On the other hand, our abundance value $(-6.20)$ is fully consistent with the determination of Smith \& Dworetsky (1993). Taking into account the presence of the spectral companion we have derived $\log \varepsilon(\mathrm{Cr} / \mathrm{H})=-6.2$ for HD 78316. This abundance value is lower than the abundance obtained by Adelman \& Pintado (2000), but higher than that published by Ryabchikova (1998). As an additional test of the accuracy of the determination of the "mean" abundances we calculated the $\mathrm{Cr}$ abundance in the star HD 175640 using 
Table 3. The average $\mathrm{Cr}$ abundance gradient $a$, its error $\sigma_{a}$ and the derived "mean" $\mathrm{Cr}$ abundance.

\begin{tabular}{|c|c|c|}
\hline Star & $\left(a \pm \sigma_{a}\right) * 10^{3}$ & $\log \varepsilon(\mathrm{Cr} / \mathrm{H})$ \\
\hline HD 1909 & $-7.5 \pm 3.3$ & $-6.28 \pm 0.15$ \\
\hline HD 33904 & $-5.0 \pm 1.1$ & $-5.91 \pm 0.06$ \\
\hline HD 49606 & $+2.0 \pm 1.4$ & $-6.20 \pm 0.04$ \\
\hline HD 71066 & $-9.3 \pm 4.1$ & $-6.23 \pm 0.10$ \\
\hline HD 78316 & $-7.8 \pm 2.3$ & $-6.20 \pm 0.14$ \\
\hline HD 110073 & $-5.6 \pm 2.2$ & $-5.66 \pm 0.05$ \\
\hline HD 124740 & $-6.2 \pm 1.6$ & $-5.71 \pm 0.14$ \\
\hline HD 165493 & $-14.2 \pm 4.5$ & $-6.82 \pm 0.15$ \\
\hline HD 168733 & $-0.3 \pm 2.1$ & $-5.59 \pm 0.09$ \\
\hline HD 175640 & $-10.5 \pm 3.1$ & $-5.58 \pm 0.03$ \\
\hline HD 178065 & $-6.2 \pm 1.6$ & $-5.99 \pm 0.05$ \\
\hline HD 196426 & $+0.4 \pm 1.9$ & $-6.66 \pm 0.05$ \\
\hline $46 \operatorname{Dra~A}^{a}$ & $-5.4 \pm 1.3$ & $-6.32 \pm 0.10$ \\
\hline 46 Dra B $^{a}$ & $-6.4 \pm 2.5$ & $-5.76 \pm 0.14$ \\
\hline
\end{tabular}

a Data from Savanov et al. (2001a).

Table 4. Cr abundance determinations in the present study and from the literature.

\begin{tabular}{llll}
\hline \hline Star & $\begin{array}{l}\log \varepsilon \\
(\mathrm{Cr} / \mathrm{H}) \\
\text { this paper }\end{array}$ & $\begin{array}{l}\log \varepsilon \\
(\mathrm{Cr} / \mathrm{H})\end{array}$ & references \\
\hline HD 1909 & -6.28 & -6.40 & Guthrie (1984) \\
& & -6.68 & Adelman et al. (1996) \\
HD 33904 & -5.91 & -5.89 & Adelman \& Pintado (2000) \\
HD 49606 & -6.20 & -5.89 & Adelman et al. (1996) \\
& & -6.20 & Smith \& Dworetsky (1993) \\
HD 78316 & -6.20 & -5.89 & Adelman \& Pintado (2000) \\
& & -6.42 & Ryabchikova (1998) \\
HD 110073 & -5.66 & -5.50 & Adelman \& Pintado (2000) \\
HD 175640 & -5.58 & -5.50 & Smith \& Dworetsky (1993) \\
HD 178065 & -5.99 & -5.9 & Guthrie (1984) \\
& & -5.84 & Pintado \& Adelman (1996) \\
& & -5.8 & Zakharova (1994) \\
HD 196426 & -6.66 & -6.60 & Smith \& Dworetsky (1993) \\
\hline
\end{tabular}

eight unblended $\mathrm{Cr}$ II lines of mult. 44 in the spectral region 4550-4620 A. Calculations were performed with the same source of $g f$-values (VALD database). We obtained the value of $\log \varepsilon(\mathrm{Cr} / \mathrm{H})$ is equal to $-5.55 \pm 0.10$, very similar to that derived from the $\mathrm{Cr}$ II lines in the far wings of the $\mathrm{H}_{\beta}$ line (-5.58).

The results presented in Fig. 1 and in Table 3 show that except for HD 49606, in all HgMn stars of our sample the Cr abundance in the atmospheres increases slightly with height in the stellar atmosphere. The average $\mathrm{Cr}$ abundance gradient $a$ is $-0.0076 \pm 0.0028$. This corresponds to an abundance increase of approximately 0.34 dex in going from the $4812.34 \AA$ line to the $4864.33 \AA$ line.

We found no vertical $\mathrm{Cr}$ stratification in the atmosphere of the HgMn star HD 49606, which is the hottest $\mathrm{HgMn}$ star in our sample. The model atmosphere parameters for this star have been taken from Adelman et al. (1996). It has been shown by Smith \& Dworetsky (1993) that the Cr II lines in the hottest

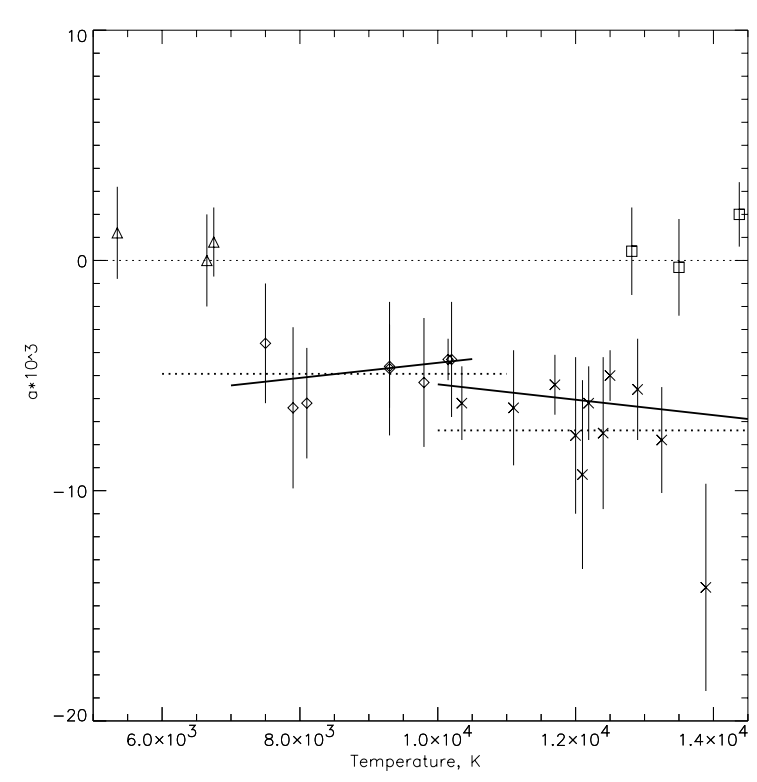

Fig. 2. Dependence of the stratification parameter $a$ on $T_{\text {eff }}$. The three points (triangles) in the "cool" end of the diagram are for the standard stars Procyon and $\iota$ Peg A and B. Diamonds denote results for Am stars and crosses are results from the current investigation of $\mathrm{HgMn}$ stars. The three open squares correspond to the stars for which no vertical stratification of the $\mathrm{Cr}$ abundance has been found. Dotted lines are mean values for the Am and $\mathrm{HgMn}$ groups, while two solid lines are the regressions for each group.

HgMn stars are intrinsically weak. We are not able to measure the chromium abundances from the Cr II lines at 4856.19, 4864.33 and $4876.40 \AA$, which are the closest to the center of the $\mathrm{H}_{\beta}$ line. However, other lines of mult. 30 indicate a constant $\mathrm{Cr}$ abundance with $\log \varepsilon(\mathrm{Cr} / \mathrm{H})=-6.20$ (Fig. 1). The star HD 49606 is of special interest because of the observation of a strong longitudinal magnetic field $(1.4 \pm 0.2 \mathrm{kG})$ reported by Glagolevskij et al. (1985). However, Hubrig \& Launhardt (1993) could not confirm the existence of such a field. The inspection of old photographic spectra revealed splitting of several lines into two components probably caused by a companion. The line profiles on our NTT spectrum show the tendency to be "square" or rectangular suggesting incipient separation into two components. Further observations at high resolution and high $S / N$ are needed to decide definitely whether HD 49606 is indeed a spectroscopic binary.

No variations of the $\mathrm{Cr}$ abundance with depth have been found for the normal late B-type star HD 196426 and the magnetic star HD 168733. The longitudinal magnetic field of HD 168733 is rather weak, about $-600 \mathrm{G}$, and not detectably variable (Mathys \& Hubrig 1997). The lack of stratification of $\mathrm{Cr}$ in the magnetic star HD 168733 is consistent with the results of the previous analyses of the stars $17 \mathrm{Com}$ and $\alpha^{2} \mathrm{CVn}$ (Savanov et al. 2001a). In contrast, an increase of the $\mathrm{Cr}$ abundance with depth was found for the cool magnetic stars $\beta \mathrm{CrB}$, $\gamma$ Equ and $10 \mathrm{Aql}$ (Savanov \& Kochukhov 1998; Savanov et al. 2001a). The result for $\gamma$ Equ has been confirmed in the later study by Ryabchikova et al. (2002) who presented also an evidence for abundance stratification of other elements $(\mathrm{Ca}, \mathrm{Fe}$, $\mathrm{Ba}, \mathrm{Si}, \mathrm{Na}, \mathrm{Pr}$ and Nd). 


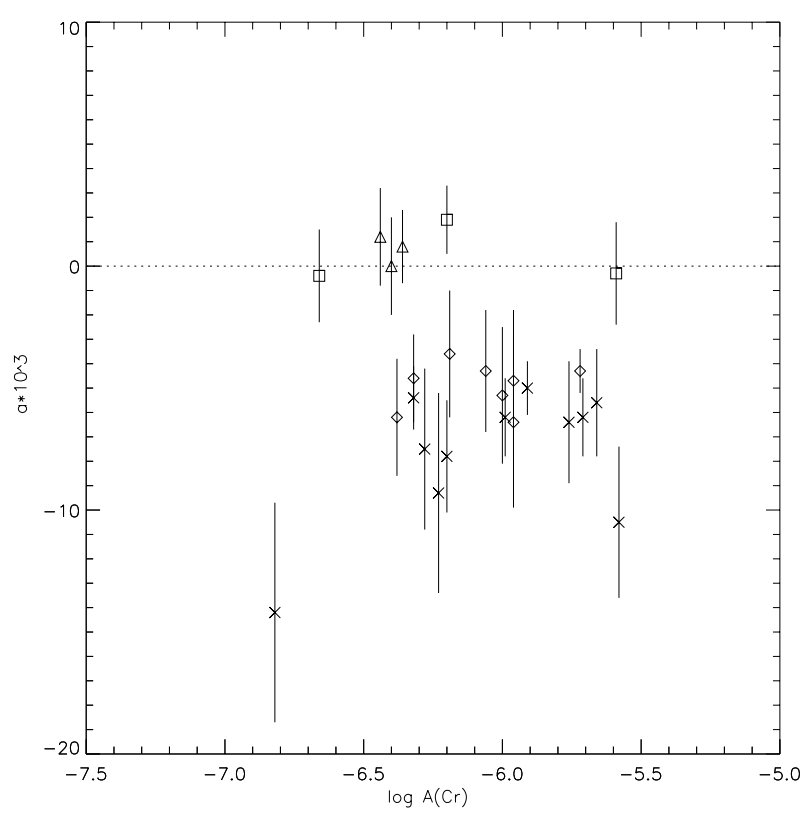

Fig. 3. Stratification parameter $a$ as a function of the $\mathrm{Cr}$ abundance. Symbols are the same as in Fig. 2.

To search for possible general relations, we studied the dependence between the vertical stratification parameter $a$ and $T_{\text {eff }}$, and between $a$ and the $\mathrm{Cr}$ abundance $\log \varepsilon(\mathrm{Cr} / \mathrm{H})$ (Figs. 2, 3). In previous studies Procyon and $\iota$ Peg A, B have been used as standards with normal atmospheres and known abundances. As $\iota$ Peg is a spectroscopic binary, spectra of the components were calculated and combined using the BINARY subprogram. As expected, no stratification of $\mathrm{Cr}$ was found in their atmospheres (Savanov \& Kochukhov 1998; Savanov et al. 2001a). These stars are presented by the three triangles in the "cool" end of Fig. 2. The diamonds show the results for Am stars which occupy the temperature region from about $7500 \mathrm{~K}$ to $10000 \mathrm{~K}$. The crosses display the results for the $\mathrm{HgMn}$ stars, whereas three open squares correspond to the stars for which no vertical stratification of $\mathrm{Cr}$ has been found (HD 49606, HD 196426 and HD 168733).

There is no significant difference in $a$ between Am and $\mathrm{HgMn}$ stars in the temperature region of about $10000 \mathrm{~K}$. From Fig. 2 we may conclude that both groups, Am and HgMn stars, show an increase of the $\mathrm{Cr}$ abundance in the upper atmospheric layers. This effect is more pronounced in stars of the $\mathrm{HgMn}$ group, for which a mean value of $a=(-7.38 \pm 0.78) \times 10^{-3}$ was obtained. For Am stars we find $a=(-4.93 \pm 0.34) \times 10^{-3}$. The mean values for both groups are shown in Fig. 2 by dotted lines. Two solid lines show the regressions for stars of each group. It is quite possible that the vertical stratification parameter $a$ depends on $T_{\text {eff }}$ in HgMn stars. To confirm this trend, more observations of $\mathrm{HgMn}$ stars on the hotter end are needed.

In Fig. 3 we plot $a$ as a function of $\log \varepsilon(\mathrm{Cr} / \mathrm{H})$. It appears that the amount of $\mathrm{Cr}$ stratification $\left(a \approx-7 \times 10^{-3}\right)$ does not depend on the average abundance, $\log \varepsilon(\mathrm{Cr} / \mathrm{H})$. One star, HD 165493, deviates strongly from this general relation. It is interesting that this hot star shows the lowest $\mathrm{Cr}$ abundance in the far wings of the $\mathrm{H}_{\beta}$ line and the strongest vertical $\mathrm{Cr}$ abundance gradient $(14.2 \pm 4.5) \times 10^{-3}$. As has been shown by
Smith \& Dworetsky (1993) the majority of HgMn stars with the $T_{\text {eff }}>13000 \mathrm{~K}$ exhibit deficiency of Cr. The most remarkable example is the star HD 186122 which is the most $\mathrm{Cr}$ deficient star known. HD 186122 has been observed with UVES in May 2000, but the extreme faintness of the Cr II lines did not allow to carry out the study of $\mathrm{Cr}$ abundances in the wings of the $\mathrm{H}_{\beta}$ line. It is possible that the radiative acceleration on $\mathrm{Cr}$ II in hotter stars is sufficient to drive it out of the atmosphere. Recently, LTE and NLTE radiative accelerations have been calculated for different elements including iron-peak elements for a stellar model of $12000 \mathrm{~K}$ by Hui-Bon-Hoa et al. (2002). They find that the abundances of the iron-peak elements that can be supported by radiation in the atmosphere are consistent with the average abundances of $\mathrm{HgMn}$ stars estimated from abundance analyses of individual stars. Additional radiative force calculations would be useful to clarify the origin of the underabundance of $\mathrm{Cr}$ in the the hotter $\left(T_{\text {eff }} \geq 13000 \mathrm{~K}\right) \mathrm{HgMn}$ stars.

\section{Conclusions}

In comparison to previous studies, our data have been acquired at much higher spectral resolution $(R \geq 70000)$ and higher signal-to-noise ratio $(S / N \geq 200)$. Such high quality data allowed us to perform an accurate study even for hotter examples of $\mathrm{HgMn}$ stars in which the Cr II lines are intrinsically weak. The results of our analysis have been compared with the previous work on Am stars and magnetic CP stars.

We found indications that there is a vertical chromium distribution in the atmospheres of nine $\mathrm{HgMn}$ stars. Since our sample of HgMn stars includes stars in a broad range of effective temperatures with different values of surface gravities and different $\mathrm{Cr}$ abundance, we took advantage of this fact to search for possible correlations between the parameters describing the vertical stratification and other stellar paramaters. The amount of $\mathrm{Cr}$ stratification in $\mathrm{HgMn}$ stars is similar to that in Am stars. Both groups, Am and HgMn stars, show an increase of chromium abundance in the upper layers of the atmosphere, but this effect is more pronounced for the stars of the HgMn group. This fact seems to support the hypothesis of a possible relationship between these two types of $\mathrm{CP}$ stars. On the other hand, the stars of these groups have in some respects very different properties. E.g. microturbulence is high in Am stars, while it is essentially zero in the $\mathrm{HgMn}$ stars (Landstreet 1998). Further studies of the Cr abundance in both types of stars are needed. In particular, it would be very enlightening to analyse in the future studies the $\mathrm{Cr}$ abundance in the spectral region before and after the Balmer jump.

As steeper slopes are found in hotter HgMn stars, it is quite possible that the vertical stratification parameter $a$ depends on $T_{\text {eff }}$. However, more observations of HgMn stars on the hotter end are needed to confirm this trend.

We found no vertical Cr stratification in the atmospheres of the normal late B-type star HD 196426, the weak magnetic late B-type star HD 168733 and the hot HgMn star HD 49606. It is likely that the hot HgMn star HD 49606 is a double-lined spectroscopis binary system and the companion should be taken into account in the abundance analysis. 
Acknowledgements. We would like to thank F. Castelli for constructive comments. Part of this work was supported by the German Deutsche Forschungsgemeinschaft, DFG project number STR645/1-1.

\section{References}

Abt, H. A., Chaffee, F. H., \& Suffolk, G. 1992, ApJ, 175, 779 Adelman, S. J. 1994, MNRAS, 266, 97

Adelman, S. J., Philip, A. G., \& Adelman, C. J. 1996, MNRAS, 282, 953

Adelman, S. J., \& Pintado, O. J. 2000, A\&A, 354, 899

Adelman, S. J., Gulliver, A. F., Kochukhov, O. P., \& Ryabchikova, T. 2002, ApJ, 575, 449

Alecian, G. 1982, A\&A, 107, 61

Dolk, L., Wahlgren, G. M., \& Hubrig, H. 2003, A\&A, submitted

Glagolevskij, J. V., Panov, K., \& Chunakova, N. M. 1985, Pisma AJ, 11,749

Guthrie, B. N. G. 1984, MNRAS, 206, 85

Hubrig, S., \& Launhardt, R. 1993, IAU Coll., 138, 350

Hubrig, S., Castelli, F., \& Wahlgren, G. M. 1999, A\&A, 346, 139

Hubrig, S., \& Castelli, F. 2001, A\&A, 375, 963

Hubrig, S., \& Mathys, G. 1995, Comments on Astrophys., 18, 167

Hui-Bon-Hoa, A., LeBlanc, F., Hauschild, P. H., \& Baron, E. 2002, A\&A, 381, 197

Khokhlova, V. L., \& Topil'skaya, G. P. 1992, Pis'ma Astron. Zh., 18, 150

Kurucz, R. L. 2000, http: //www . cfaku5 . harvard.edu

Kurucz, R. L., \& Bell, B. 1995, Atomic Line List, CD-ROM No. 23, Smithonian Astroph. Obs. Cambridge, MA
Landstreet, J. D. 1998, A\&A, 338, 1041

Lanz, T., Artru, M. C., Didelon, P., \& Mathys, G. 1993, A\&A, 272, 465

Mathys, G., \& Hubrig, S. 1997, A\&AS, 124, 475

Pintado, O. I., \& Adelman, S. J. 1996, A\&AS, 118, 283

Piskunov, N. 1992, in Stellar magnetism, ed. Y. V. Glagolevskij, \& I. I. Romanyuk (Nauka, St. Peterburg), 92

Piskunov, N., Kupka, F., Ryabchikova, T. A., et al. 1995, A\&AS, 112, 525

Ryabchikova, T., Kotchoukhov, O., Galazutdinov, G., Musaev, F., \& Adelman, S. J. 1998, Contributions Astronomical Observatory Skalnate Pleso, 27, 258

Ryabchikova, T. 1998, Contributions Astronomical Observatory Skalnate Pleso, 27, 319

Ryabchikova, T., Piskunov, N., Kochukhov, O., et al. 2002, A\&A, 384, 545

Ryabchikova, T., Wade, G. A., \& LeBlanc, F. 2003, A\&A, in press

Savanov, I., \& Kochukhov, O. 1998, Astron. Lett., 24, 516

Savanov, I. S., Kochukhov, O. P., \& Tsymbal, V. V. 2001a, Astrophys., 44,64

Savanov, I. S., Kochukhov, O. P., \& Tsymbal, V. V. 2001b, Astrophys., 44, 206

Smith, K. C., \& Dworetsky, M. M. 1993, A\&A, 274, 335

Tsymbal, V. V. 1996, in Model Atmospheres and Stellar Spectra, ed. S. J. Adelman, F. Kupka, \& W. W. Weiss, ASP Conf. Ser., 198

Woolf, V. M., \& Lambert, D. L. 1999, ApJ, 521, 414

Wahlgren, G. M., Ilyin, I., \& Kochukhov, O. 2001, AAS, 199, 3504

Wahlgren, G. M., Hubrig, S., \& Dolk, L. 2002, IBVS, 5290, 1

Zakharova, L. A. 1994, AZh, 38, 520 